Pacific

Journal of

Mathematics

THE STRAIGHTENING THEOREM FOR TANGENT-LIKE MAPS

Piotr GALĄZKa AND JANinA Kotus 


\title{
THE STRAIGHTENING THEOREM FOR TANGENT-LIKE MAPS
}

\author{
PiOTR GALAZZKA AND JANinA Kotus
}

\begin{abstract}
By analogy to polynomial-like maps, we introduce a notion of tangent-like maps. The main result of this paper is the straightening theorem. It says that a tangent-like map is quasiconformally equivalent to some tangent-type function $f: \mathbb{C} \rightarrow \overline{\mathbb{C}} \backslash\{a, b\}$ for $a \neq b$, which is unique up to an affine map. We also prove that quasiconformal conjugacy is conformal on the interior of the filled Julia set.
\end{abstract}

\section{Introduction}

In [1985], A. Douady and J. H. Hubbard introduced a notion of the polynomial-like maps. A polynomial-like map of degree $d \geq 2$ is a triple $(f, U, V)$ where $U$ and $V$ are open subsets of $\mathbb{C}$ isomorphic to discs, with $U$ relatively closed in $V$ and $f: U \rightarrow V$ an analytic map, proper of degree $d$. Of course, every polynomial of degree $d \geq 2$ is locally polynomial-like of the same degree. It appears that some transcendental meromorphic functions are locally polynomial-like of finite degree (for example, $f(z)=\cos (z)-2$ in some neighborhood of zero). Small perturbations of polynomial-like maps of degree $d$ stay in the same class of maps. The filled Julia set of a polynomial-like map $f: U \rightarrow V$ is defined as $K(f)=\bigcap_{n \geq 0} f^{-n}(U)$. The set $K(f)$ is a compact subset of $U$. Douady and Hubbard proved the straightening theorem [1985, Theorem 1], which says that every polynomial-like map $f: U \rightarrow V$ is hybrid equivalent to a polynomial of degree $d$. This result has many applications, mainly in the renormalization theory of rational functions initiated by D. Sullivan. For other applications see [McMullen 2000].

Nevertheless, the concept of polynomial-like maps does not fit with those meromorphic functions whose inverses have at least one transcendental singularity at some point in $\mathbb{C}$ (for example, for $\arctan (z)$ at $\pm i, \log (z)$ at 0 , and so on).

MSC2000: primary 30D05; secondary 37F30.

Keywords: meromorphic functions, Julia set, filled Julia set, polynomial-like maps, hybrid equivalent.

Kotus is partially supported by a grant Chaos, fraktale i dynamika konforemna N N201 022233 and by EU FP6 Marie Curie Tok SPADE2 at IMPAN. 
The aim of this paper is to introduce a definition of tangent-like maps and prove an analogue of the Straightening Theorem for this class.

The paper is organized as follows. In Section 2, we describe the dynamics of some family of transcendental meromorphic functions with two omitted values. In Section 3, we introduce a notion of tangent-like maps. In Section 4, we prove the main result of our paper, that is, the straightening theorem.

\section{A model family}

The Fatou set $F(f)$ of a meromorphic function $f: \mathbb{C} \rightarrow \overline{\mathbb{C}}$ is defined in exactly the same manner as for rational functions: $F(f)$ is the set of points $z \in \mathbb{C}$ such that all the iterates are defined and form a normal family on a neighborhood of $z$. The Julia set $J(f)$ is the complement of $F(f)$ in $\overline{\mathbb{C}}$. Thus, $F(f)$ is open, $J(f)$ is closed, $F(f)$ is completely invariant while $f^{-1}(J(f)) \subset J(f)$, and $f(J(f) \backslash\{\infty\})=$ $J(f)$. For a general description of the dynamics of meromorphic functions see for example [Bergweiler 1993]. It follows from Montel's criterion of normality that if $f: \mathbb{C} \rightarrow \overline{\mathbb{C}}$ has at least one pole that is not an omitted value, then

$$
J(f)=\overline{\bigcup_{n \geq 0} f^{-n}(\infty)} .
$$

See [Baker et al. 1991b]. Let $\operatorname{Crit}(f)=\left\{z \in \mathbb{C}: f^{\prime}(z)=0\right\}$ be the set of critical points of $f$. We note that we do not consider multiple poles as critical points. Let $\operatorname{Sing}\left(f^{-1}\right)$ denote the set of singular values of $f$, that is, $c \in \operatorname{Sing}\left(f^{-1}\right)$ if $c \in \mathbb{C}$ and $c$ is a critical or an asymptotic value of $f$.

Consider a family of transcendental meromorphic functions of the form

$$
T_{\beta}(z)=\frac{e^{z / 2}-e^{\beta z / 2}}{e^{z / 2}-\beta e^{\beta z / 2}}
$$

for $\beta \in \mathbb{C} \backslash\{0,1\}$. It is easy to see that $T_{\beta}(z)$ has no critical points. It has two omitted values 1 and $1 / \beta$ (which are always the asymptotic values), and its poles are simple. Thus $T_{\beta}: \mathbb{C} \rightarrow \overline{\mathbb{C}} \backslash\{1,1 / \beta\}$ is a regular holomorphic cover if we define a derivative of $f$ by the means of the spherical metric. One can easily check for all $\beta \in \mathbb{C} \backslash\{0,1\}$ that $z_{0}=0$ is an attracting fixed point, that is,

$$
T_{\beta}(0)=0 \quad \text { and } \quad T_{\beta}^{\prime}(0)=1 / 2,
$$

where $T_{\beta}^{\prime}$ denotes a derivative of $T_{\beta}$ defined with respect to the Euclidean metric. We note that the choice of a multiplier of the attracting fixed point is arbitrary. It could be any complex number $\alpha$ such that $0<|\alpha|<1$.

The next proposition shows why the family of functions $T_{\beta}$ is called a model family. 
Proposition 2.1. For every $\beta \in \mathbb{C} \backslash\{0,1\}$ the map $T_{\beta} \circ A$, in which $A(z)=$ $z+4 k \pi i /(1-\beta)$ for $k \in \mathbb{Z}$, is a regular holomorphic covering $f$ from $\mathbb{C}$ onto $\overline{\mathbb{C}} \backslash\{1,1 / \beta\}$ such that $f(0)=0$ and $f^{\prime}(0)=1 / 2$.

Proof. Suppose that $f: \mathbb{C} \rightarrow \overline{\mathbb{C}} \backslash\{1,1 / \beta\}$ is an another regular covering. Then there exists a Möbius map $A: \mathbb{C} \rightarrow \mathbb{C}$ such that $f=T_{\beta} \circ A$. Then $A(z)=a z+b$ for $a \neq 0$, and

$$
f(z)=T_{\beta}(a z+b)=\frac{e^{(a z+b) / 2}-e^{\beta(a z+b) / 2}}{e^{(a z+b) / 2}-\beta e^{\beta(a z+b) / 2}} .
$$

It implies

$$
0=f(0)=T_{\beta}(b)=\frac{e^{(1-\beta) b / 2}-1}{e^{(1-\beta) b / 2}-\beta}
$$

if and only if $b=4 k \pi i /(1-\beta)$ for $k \in \mathbb{Z}$. But $\frac{1}{2}=f^{\prime}(0)=T_{\beta}^{\prime}(b) A^{\prime}(0)=\frac{1}{2} a$; thus $a=1$. Therefore $f(z)=T_{\beta}(z+4 k \pi i /(1-\beta))$.

It follows from the classification of the periodic components of the Fatou set (see [Baker et al. 1991a]) that at least one of the asymptotic values $\{1,1 / \beta\}$ must belong to the basin of attraction $B(0)$ of $z_{0}=0$. It is easy to see that for large $\beta$ the asymptotic value $1 / \beta$ is close to $z_{0}=0$. Now we show that, for sufficiently large $\beta$, the asymptotic value $1 / \beta \in B(0)$. We rewrite $T_{\beta}(z)$ as

$$
T_{\beta}(z)=1+\frac{\beta e^{z(\beta-1) / 2}-e^{z(\beta-1) / 2}}{1-\beta e^{z(\beta-1) / 2}} .
$$

For a given $\beta \in \mathbb{C} \backslash\{0,1\}$, we choose disc $D(0,3 /|2 \beta|)$. We want to prove that for large $\beta$

$$
\overline{T_{\beta}(D(0,3 /|2 \beta|))} \subset D(0,3 /|2 \beta|) .
$$

Let $\beta=|\beta| e^{i \alpha}$. We consider $z=3 e^{i \theta} /|2 \beta|$ for $\theta \in[0,2 \pi)$. Then

$$
\frac{z(\beta-1)}{2}=\frac{3}{2} \frac{e^{i \theta}}{|\beta|} \frac{\left(|\beta| e^{i \alpha}-1\right)}{2}=\frac{3}{4} e^{i(\theta+\alpha)}-\frac{3}{4} \frac{e^{i \theta}}{|\beta|} .
$$

For sufficiently large $\beta$ we can approximate $z(\beta-1) / 2$ by $\frac{3}{4} e^{i(\theta+\alpha)}$. Observe that for $\theta=-\alpha$, we have $\frac{3}{4} e^{i(\theta+\alpha)}=\frac{3}{4}$, while for $\theta=\pi-\alpha$ we have $\frac{3}{4} e^{i(\theta+\alpha)}=-\frac{3}{4}$. Consequently

$$
\begin{aligned}
T_{\beta}\left(\frac{3}{2} \frac{e^{-i \alpha}}{|\beta|}\right) & =1+\frac{\beta e^{3 / 4}-e^{3 / 4}}{1-\beta e^{3 / 4}}=1+\frac{e^{3 / 4}(\beta-1)}{1-\beta e^{3 / 4}} \\
& =1-\frac{e^{3 / 4}(\beta-1)}{e^{3 / 4}\left(\beta-e^{-3 / 4}\right)}=\frac{1-e^{-3 / 4}}{\beta-e^{-3 / 4}} \approx \frac{1}{2(\beta-0.5)} .
\end{aligned}
$$


Analogously

$$
\begin{aligned}
T_{\beta}\left(\frac{3}{2} \frac{e^{i(\pi-\alpha)}}{|\beta|}\right) & =1+\frac{\beta e^{-3 / 4}-e^{-3 / 4}}{1-\beta e^{-3 / 4}}=1+\frac{e^{-3 / 4}(\beta-1)}{1-\beta e^{-3 / 4}} \\
& =1-\frac{e^{-3 / 4}(\beta-1)}{e^{-3 / 4}\left(\beta-e^{3 / 4}\right)}=\frac{1-e^{3 / 4}}{\beta-e^{3 / 4}} \approx-\frac{1}{\beta-2} .
\end{aligned}
$$

Consequently, for $z \in \partial D(0,3 /|2 \beta|)$ the maximum of $\left|T_{\beta}(z)\right| \leq 1 /|\beta-2|$. Enlarging $\beta$ if necessary, we may say that $1 /|\beta-2|<3 e^{i \theta} /|2 \beta|$. Thus we may claim that (1) is satisfied. It proves that $D(0,3 /|2 \beta|) \subset B(0)$. Since $1 / \beta \in D(0,3 /|2 \beta|)$, we have $1 / \beta \in B(0)$.

From now we assume that $\beta$ is so large that $1 / \beta \in B(0)$.

Let $D$ be a component of the Fatou set $F\left(T_{\beta}\right)$ containing $z_{0}=0$. Then $D$ is forward invariant. Of course $D \subset B(0)$ and $1 / \beta \in D$. Take a component $D_{0}$ of $T_{\beta}^{-1}(D)$ containing a fixed point $z_{0}=0$. Since $1 / \beta$ is an omitted value, $D_{0}$ must contain an asymptotic tract corresponding to $1 / \beta$. Thus $D$ must be a completely invariant unbounded domain and $D=B(0)$. As $B(0)$ is completely invariant, $J\left(T_{\beta}\right)=\partial B(0)$. One can easily prove that $B(0)$ is simply connected if and only if the second asymptotic value $1 \notin B(0)$. By analogy to polynomials we define the filled Julia set

$$
K\left(T_{\beta}\right)=\left\{z \in \mathbb{C}: T_{\beta}^{n}(z) \notin \overline{B(0)} \text { for all } n \in \mathbb{N} \cup\{0\}\right\} .
$$

Then the Julia set can be written as

$$
J\left(T_{\beta}\right)=\partial K\left(T_{\beta}\right) \cup\{\infty\} .
$$

Observe that the interior of $K\left(T_{\beta}\right)$ is nonempty if and only if the second asymptotic value 1 is related to some periodic component of the Fatou set $F\left(T_{\beta}\right)$ different from $B(0)$. Since $\operatorname{Sing}\left(T_{\beta}^{-1}\right)$ consists of two asymptotic values $\{1,1 / \beta\}$, the map $T_{\beta}$ belongs to the class $\mathscr{S}$ defined in [Bergweiler 1993]. Then the classification of periodic components of the $F\left(T_{\beta}\right)$ is the same as for rational map. That is, $T_{\beta}$ does not have Baker domains; see [Bergweiler 1993]. Moreover, $T_{\beta}$ does not have Herman rings. The proof is same for tangent family; see [Keen and Kotus 1997]. Thus the interior $K\left(T_{\beta}\right)$ is nonempty if $F\left(T_{\beta}\right)$ has either some attracting periodic cycle, some parabolic periodic cycle, or some Siegel periodic point. In the last case $1 \in J\left(T_{\beta}\right)$.

Finally we would like to explain why we prefer to work with the family $T_{\beta}(z)$ instead of the tangent family $f_{\lambda}(z)=\lambda \tan (z)$ with $\lambda \in \mathbb{C}^{*}$, which was described [Keen and Kotus 1997]. The map $f_{\lambda}$ is a regular holomorphic cover of $\overline{\mathbb{C}} \backslash\{ \pm \lambda i\}$ and the orbits of two asymptotic values $\pm \lambda i$ are symmetric - they belong either to the same or to two symmetric periodic cycles of the Fatou set; see [Keen and Kotus 1997]. To get some analogy with quadratic polynomials, we should have 
an attracting fixed point and associated with it some asymptotic value, while the second asymptotic value should be "free". But this never happens in the classical tangent family. On the other hand, the proposed family admits phenomena discovered in [Keen and Kotus 1997] for tangent maps, for example, collisions of repelling periodic points with poles, if some iterate of the asymptotic value 1 is eventually mapped onto a pole.

\section{Tangent-like maps}

Definition 3.1. The triple $(f, U, V)$ is a tangent-like map if

(i) $U$ and $V$ are Jordan domains such that $\bar{U} \subset V$,

(ii) there exist $v \in V$ and $u \in \partial U$ such that $f: \bar{U} \backslash\{u\} \rightarrow \bar{V} \backslash\{v\}$ is a cover,

(iii) $f$ is holomorphic on $U$, and

(iv) $U$ is a quasidisc.

Here $u$ plays a role of essential singularity, and $v$ is an asymptotic value of $f$.

Definition 3.2. The set

$$
K(f, U, V):=\bigcap_{n \geq 0} \overline{f^{-n}(U)}
$$

is called the filled Julia set of $(f, U, V)$, while $J(f, U, V):=\partial K(f, U, V)$ is called the Julia set of $(f, U, V)$.

Proposition 3.3. For every sufficiently large $\beta \in \mathbb{C} \backslash\{0,1\}$, the function $T_{\beta}(z)$ is a tangent-like map.

Proof. We know that if $\beta$ sufficiently large, the asymptotic value $1 / \beta$ belongs to the basin of the attraction of the fixed point $z_{0}=0$. Since $T_{\beta}$ is locally linearizable in some disc $D_{0}:=D(0, \varepsilon)$ (shrinking $\varepsilon$ if necessary) we can say that there exist a quasidisc $D_{0}$ and an integer $N \in \mathbb{N}$ such that

$$
\begin{aligned}
& 0 \in D_{0}, \\
& D_{0} \subset \mathbb{C} \backslash K\left(T_{\beta}\right) \text {, } \\
& T_{\beta}\left(D_{0}\right) \subset D_{0}, \\
& T_{\beta}^{N}(1 / \beta) \in D_{0}, \\
& T_{\beta}^{N-1}(1 / \beta) \notin \bar{D}_{0}, \\
& T_{\beta}^{N}(1) \notin \bar{D}_{0} .
\end{aligned}
$$

Let $D_{i+1}$ denotes a component of $T^{-1}\left(D_{i}\right)$ that contains $D_{i}$ for $i=0, \ldots, N-1$. Then $D_{N}$ contains an asymptotic value $1 / \beta$, and a branch of the inverse function $T_{\beta}^{-1}$ that maps $D_{N} \backslash\{1 / \beta\}$ onto $D_{N+1}$ has a logarithmic singularity. Thus $D_{N+1}$ is a simply connected unbounded domain, and $T_{\beta}: D_{N+1} \rightarrow D_{N} \backslash\{1 / \beta\}$ is a universal cover. Let $U:=\mathbb{C} \backslash \bar{D}_{N+1}$ and $V:=\mathbb{C} \backslash \bar{D}_{N}$. Set also $u:=\infty$ and $v=1$. Then $T_{\beta}: \bar{U} \backslash\{u\} \rightarrow \bar{V} \backslash\{v\}$ is a universal cover. Since $D_{0}$ is a quasidisc, $D_{N+1}$ and $U$ are also quasidiscs. It proves that $\left(T_{\beta}, U, V\right)$ is a tangent-like map. 
Definition 3.4. We say that two tangent-like maps $\left(f_{1}, U_{1}, V_{1}\right)$ and $\left(f_{2}, U_{2}, V_{2}\right)$ are hybrid equivalent if there exists a quasiconformal homeomorphism $\phi$ mapping a neighborhood of $K\left(f_{1}, U_{1}, V_{1}\right)$ onto a neighborhood of $K\left(f_{2}, U_{2}, V_{2}\right)$ in $\mathbb{C}$ such that $\phi \circ f_{1}(z)=f_{2} \circ \phi$ on a neighborhood of $K\left(f_{1}, U_{1}, V_{1}\right)$ and $(\partial \phi / \partial z)(z)=0$ for $z \in K\left(f_{1}, U_{1}, V_{1}\right)$.

\section{The straightening theorem}

Definition 4.1. Suppose that $\gamma$ is a Jordan curve. A map $p: \mathbb{R} \rightarrow \gamma$ is called a unit speed parametrization of $\gamma$ if for all $t \in \mathbb{R}, p^{\prime}(t)$ is well defined, $\left|p^{\prime}(t)\right|=1$, and $p(t)$ winds anticlockwise as $t$ increases.

Definition 4.2. If $\gamma_{1}$ and $\gamma_{2}$ are two Jordan curves, $p_{1}$ and $p_{2}$ are their respective unit speed parametrizations. Let $f: \gamma_{1} \rightarrow \gamma_{2}$ be a homeomorphism. Then there exists a lift $\tilde{f}: \mathbb{R} \rightarrow \mathbb{R}$ such that $f \circ p_{1}=p_{2} \circ \tilde{f}$. We say that $f$ is a $C^{r}$ diffeomorphism with respect to the parametrization if and only if $\tilde{f}$ is a $C^{r}$ diffeomorphism.

We leave to the reader a proof of the next lemma.

Lemma 4.3. Let $D \subset \mathbb{C}$ be a Jordan domain such that $\partial D=\gamma\left(S^{1}\right)$ and $\gamma$ is $C^{2}$ in some neighborhood of $S^{1}$. Then $\partial D$ has a constant speed parametrization. If $\phi: \mathbb{D} \rightarrow D$ is a conformal isomorphism with homeomorphic extension $\tilde{\phi}: \overline{\mathbb{D}} \rightarrow$ $\bar{D}$, then the restriction $\tilde{\phi}: S^{1} \rightarrow \partial D$ is a $C^{1}$ diffeomorphism with respect to the parametrization.

For a given conformal annulus $A \subset \mathbb{C}$ such that each component of the boundary is a Jordan curve, let $\partial A^{+}$and $\partial A^{-}$denote respectively the outer and the inner component. Let $A_{1}$ and $A_{2}$ be two annuli. We say that a homeomorphism $h$ : $\partial A_{1} \rightarrow A_{2}$ is sense preserving if $h: \partial A_{1}^{+} \rightarrow \partial A_{2}^{+}$and $h: \partial A_{1}^{-} \rightarrow \partial A_{2}^{-}$are sense preserving homeomorphisms.

Let

$$
\mathbb{A}_{t}:=\left\{z \in \mathbb{C}: 1<|z|<e^{t}\right\} \text { for } t>0 .
$$

Lemma 4.4. Let $\phi: \partial \mathbb{A}_{1} \rightarrow \partial \mathbb{A}_{1}$ be a sense preserving homeomorphism such that its restriction to components of $\partial A_{1}$ is a $C^{1}$ diffeomorphism with respect to the parametrization. Then there exists a homeomorphic extension $\phi: \overline{\mathbb{A}}_{1} \rightarrow \overline{\mathbb{A}}_{1}$ that is quasiconformal on $\mathbb{A}_{1}$.

Proof. There must exist a $C^{1}$ lift of $\phi$ that commutes with the translation $T(x)=$ $x+2 \pi$. Suppose that $a: \mathbb{R} \rightarrow \mathbb{R}$ is a lift of $\left.\phi\right|_{\partial A^{-}}$and $b$ is a lift of $\left.\phi\right|_{\partial A^{+}}$, with $a$ and $b$ both increasing maps. Then $\phi\left(e^{i} t\right)=e^{i a(t)}$ and $\phi\left(e^{1+i t}\right)=e^{1+i b(t)}$ for all $t \in \mathbb{R}$. Then the interpolation $\phi\left(e^{s+i t}\right):=e^{(1-s) i a(t)+s(1+i b(t))}$ is quasiconformal.

Lemma 4.5. Let $A_{1}$ and $A_{2} \subset$ be two annuli whose boundary components are images of $S^{1}$ by $C^{2}$ diffeomorphisms. Let $h: \partial A_{1} \rightarrow \partial A_{2}$ be a sense preserving 
homeomorphism that extends to a $C^{2}$ diffeomorphism on a neighborhood of $\partial A_{1}$. Then there exists a homeomorphic extension $h: \bar{A}_{1} \rightarrow \bar{A}_{2}$ that is quasiconformal on $A_{1}$

Proof. For $t>0$, the map $L_{t}: \mathbb{C}^{*} \rightarrow \mathbb{C}^{*}$ defined as $L_{t}\left(r e^{i t}\right)=r^{t} e^{i t}$ is a real-analytic quasiconformal homeomorphism. It maps $\mathbb{A}_{1}$ homeomorphically onto $\mathbb{A}_{t}$. For $i=1,2$, choose a conformal isomorphism $\phi_{i, 1}$ from the interior of $\partial A_{i}^{+}$to $\mathbb{D}$, and let $A_{i, 1}:=\phi_{i, 1}\left(A_{i}\right)$. Next, choose a conformal isomorphism $\phi_{i, 2}$ from the exterior of $\partial A_{i}^{-}$to $\overline{\mathbb{C}} \backslash \mathbb{D}$, and let $A_{i, 2}:=\phi_{i, 2}\left(A_{i, 1}\right)$. Finally choose a conformal isomorphism $\phi_{i, 3}$ from $A_{i, 2}$ to $\mathbb{A}_{t_{i}}$ for some $t_{i}>0$. (Notice that the boundary components of $A_{i, 2}$ are both conformal images of $S^{1}$, so by the Schwartz reflection principle, $\phi_{i, 3}$ extends holomorphically to a neighborhood of the closure of $A_{i, 2}$.) Now define $\phi_{i}: A_{i} \rightarrow \mathbb{A}_{1}$ by $\phi_{i}:=L_{1 / t_{i}} \circ \phi_{i, 3} \circ \phi_{i, 2} \circ \phi_{i, 1}$. This is a conformal isomorphism that extends holomorphically to a map $\phi_{i}: \bar{A}_{i} \rightarrow \overline{\mathbb{A}}_{1}$. We can define $H: \partial \mathbb{A}_{1} \rightarrow \partial \mathbb{A}_{1}$ by $H=\phi_{2} \circ h \circ \phi_{1}$. This is a sense preserving homeomorphism. It follows from Lemma 4.3 and the Schwartz reflection principle that $H$ restricts on the boundary components of $\partial \mathbb{A}_{1}$ to give $C^{1}$ diffeomorphic maps with respect to parametrization. By Lemma 4.4, $H$ admits a homeomorphic extension of $H: \overline{\mathbb{A}}_{1} \rightarrow \overline{\mathbb{A}}_{1}$ that is quasiconformal on $\mathbb{A}_{1}$. This induces an extension $h: \bar{A}_{1} \rightarrow \bar{A}_{2}$.

Lemma 4.6. If $D$ is a quasidisc and $\phi: \mathbb{D} \rightarrow D$ is conformal, then $\phi$ has a quasiconformal extension $\bar{\phi}: \overline{\mathbb{C}} \rightarrow \overline{\mathbb{C}}$ that is real analytic on $\overline{\mathbb{C}} \backslash \overline{\mathbb{D}}$.

Proof. Suppose that $h: \overline{\mathbb{C}} \rightarrow \overline{\mathbb{C}}$ is a quasiconformal homeomorphism such that $h(\mathbb{D})=D$. Consider a reflection $j: \overline{\mathbb{C}} \rightarrow \overline{\mathbb{C}}$ in the unit circle. Let $\psi: \overline{\mathbb{C}} \backslash \overline{\mathbb{D}} \rightarrow$ $\overline{\mathbb{C}} \backslash \overline{\mathbb{D}}$ be a conformal isomorphism. Then $\phi$ and $\psi$ has a homeomorphic extension to $\overline{\mathbb{D}}$ and $\overline{\mathbb{C}} \backslash \mathbb{D}$. Thus $h^{-1} \circ \phi: S^{1} \rightarrow S^{1}$ is a homeomorphism. Define a map $H=j \circ \psi^{-1} \circ h \circ j \circ h^{-1} \circ \phi$. Then $H$ is a quasiconformal extension of $h^{-1} \circ \phi$ to $\overline{\mathbb{D}}$. Then $h^{-1} \circ \phi$ must be quasisymmetric. If follows from [Douady and Earle 1986] that $h^{-1} \circ \phi$ has a real analytic extension $E_{h^{-1} \circ \phi}: \mathbb{D} \rightarrow \mathbb{D}$. Take $\tilde{\phi}$ on $\overline{\mathbb{D}}$ and $\tilde{\phi}:=\psi \circ j \circ E_{h^{-1} \circ \phi} \circ j$ on $\mathbb{C} \backslash \mathbb{D}$.

Proposition 4.7. Suppose that $\left(f_{1}, U_{1}, V_{1}\right)$ and $\left(f_{2}, U_{2}, V_{2}\right)$ are two tangent-like maps. Then there exists a quasiconformal homeomorphism $\phi: \bar{V}_{1} \rightarrow \bar{V}_{2}$ such that $f_{2} \circ \phi(z)=\phi \circ f_{1}(z)$ for $z \in \bar{U}_{1} \backslash\left\{u_{1}\right\}$ and such that $\phi$ is holomorphic on $U_{1}$.

Proof. For simplicity, we may assume that $V_{1}=V_{2}=\mathbb{D}$ and $v_{1}=v_{2}=0$. To get a general case one must conjugate $f_{1}$ and $f_{2}$ with Riemann maps. Define $A_{i}=V_{i} \backslash \bar{U}_{i}$ for $i=1,2$. Since $f_{1}$ and $f_{2}$ are universal coverings of $\mathbb{D}^{*}$, there must exist a conformal isomorphism $h: U_{1} \rightarrow U_{2}$ such that

$$
f_{2} \circ h(z)=f_{1}(z) \text { for } z \in U_{1} .
$$


Since $U_{1}, U_{2}$ are quasidiscs there exist an extension $\tilde{h}: \bar{U}_{1} \rightarrow \bar{U}_{2}$ such that $f_{2} \circ$ $\tilde{h}(z)=f_{1}(z)$ for $z \in \partial U_{1} \backslash\left\{u_{1}\right\}$. We want to construct a quasiconformal homeomorphism $H: A_{1} \rightarrow A_{2}$ such that $H(z)=\operatorname{Id}(z)$ if $z \in \partial V_{1}$ and $H(z)=\tilde{h}(z)$ for $z \in \partial U_{1}$. Let's take two conformal isomorphisms $g_{i}: \mathbb{D} \rightarrow U_{i}$ for $i=1,2$ such that $g_{2} \circ g_{1}^{-1}=h$. Lemma 4.6 implies that $g_{i}$ extends to a quasiconformal self-map of $\overline{\mathbb{C}}$ that is real-analytic on $\overline{\mathbb{C}} \backslash \overline{\mathbb{D}}$. Let $\tilde{A}_{i}:=g_{i}^{-1}\left(A_{i}\right)$ for $i=1,2$ be two annuli. By Lemma 4.5, there exists a quasiconformal homeomorphism $\tilde{\phi}: \tilde{A}_{1} \rightarrow \tilde{A}_{2}$ that agrees with $g_{2}^{-1} \circ \tilde{h} \circ g_{1}=\operatorname{Id}$ on $\partial \tilde{A}_{1}^{-}$and with $g_{2}^{-1} \circ \operatorname{Id} \circ g_{1}$ on $\partial \tilde{A}_{1}{ }^{+}$. Then $\phi:=g_{2} \circ \tilde{\phi} \circ g_{1}^{-1}$ is a quasiconformal homeomorphism agreeing with $\tilde{h}$ on $\partial A_{1}{ }^{-}$ and with Id on $\partial A_{1}{ }^{+}$. Thus $\phi \circ f_{1}=f_{2} \circ \phi$ on $\partial U_{1} \backslash\left\{u_{1}\right\}$. We extend $\phi$ to $U_{1}$ by setting $\phi=h$.

Theorem 4.8. Every tangent-like map is hybrid equivalent to some $T_{\beta} \circ A$ with sufficiently large $\beta \in \mathbb{C} \backslash\{0,1\}$ and $A(z)=z+4 k \pi i /(1-\beta)$ for $k \in \mathbb{Z}$.

Proof. It follows from Proposition 3.3 that we can choose a map $T_{\beta}$ having a tangent-like restriction $\left(F_{0}, U_{0}, V_{0}\right)$. This is possible if $\beta$ is sufficiently large. Then the asymptotic value $1 / \beta$ belongs to the basin of attraction of $z_{0}=0$. Let $(f, U, V)$ be a tangent-like map. It follows from Proposition 4.7 that there is a homeomorphism $\phi: \bar{V}_{0} \rightarrow \bar{V}$ that is quasiconformal on $V_{0}$ and holomorphic on $U_{0}$ and that has $\phi \circ F_{0}(z)=f \circ \phi(z)$ for $z \in \bar{U}_{0} \backslash\left\{u_{0}\right\}$. Now we define a map $F(z):=\phi^{-1} \circ f \circ \phi(z)$ for $z \in U_{0}$ and $F(z)=T_{\beta}(z)$ if $z \notin U_{0}$. Then $F$ is well defined in $\mathbb{C}$ and holomorphic outside the closure of $F^{-1}\left(V_{0} \backslash \bar{U}_{0}\right)$. For any $z \in \mathbb{C}$, the forward trajectory $F^{n}(z)$ lands at most once in $F^{-1}\left(V_{0} \backslash \bar{U}_{0}\right)$. Let $\sigma_{0}$ denote the standard conformal structure on $\overline{\mathbb{C}}$. We define a new structure $\sigma$ by $\sigma=\left(F^{n}\right)^{*} \sigma_{0}$ on $F^{-n}\left(V_{0} \backslash U_{0}\right)$ for $n \geq 1$, by $\sigma=\sigma_{0}$ on $K(F)=\phi^{-1}(K(f))$, and by $\sigma=\sigma_{0}$ on $\overline{\mathbb{C}} \backslash U_{0}$. The structure $\sigma$ is $F$ invariant, that is, $F^{*}(\sigma)=\sigma$. There exists a quasiconformal homeomorphism $\Phi: \overline{\mathbb{C}} \rightarrow \overline{\mathbb{C}}$ fixing $0,1, \infty$ and such that $\Phi^{*}(\sigma)=\sigma_{0}$; see [Boyarskiu 1955]. Then

$$
g=\Phi \circ F \circ \Phi^{-1}: \mathbb{C} \rightarrow \overline{\mathbb{C}} \backslash\{1, w\}
$$

for some $w \neq 1$. Since $\sigma$ agrees with $\sigma_{0}$ on a neighborhood of 0 (more precisely on $\left.\mathbb{C} \backslash U_{0}\right), \Phi$ is holomorphic on this neighborhood, and $g^{\prime}(0)=F_{0}^{\prime}(0)=1 / 2$. So $g$ is a regular holomorphic covering from $\mathbb{C}$ onto $\overline{\mathbb{C}} \backslash\{1, w\}$. By Proposition 2.1, $g=T_{\beta} \circ A$, where $A(z)=z+4 k \pi i /(1-\beta)$ for $k \in \mathbb{Z}$ and $\beta=1 / w$.

\section{Acknowledgments}

We are very grateful to the referee for constructive remarks and suggestions, which influenced the final exposition of our work. 


\section{References}

[Baker et al. 1991a] I. N. Baker, J. Kotus, and Y. N. Lü, "Iterates of meromorphic functions, III: Preperiodic domains", Ergodic Theory Dynam. Systems 11:4 (1991), 603-618. MR 92m:58115 Zbl 0774.30023

[Baker et al. 1991b] I. N. Baker, J. Kotus, and L. Yinian, "Iterates of meromorphic functions, I", Ergodic Theory Dynam. Systems 11:2 (1991), 241-248. MR 92m:58113 Zbl 0711.30024

[Bergweiler 1993] W. Bergweiler, "Iteration of meromorphic functions", Bull. Amer. Math. Soc. (N.S.) 29:2 (1993), 151-188. MR 94c:30033 Zbl 0791.30018

[Boyarskiı̌ 1955] B. V. Boyarskiu,, "Homeomorphic solutions of Beltrami systems", Dokl. Akad. Nauk SSSR (N.S.) 102 (1955), 661-664. In Russian. MR 17,157a

[Douady and Earle 1986] A. Douady and C. J. Earle, "Conformally natural extension of homeomorphisms of the circle”, Acta Math. 157:1-2 (1986), 23-48. MR 87j:30041 Zbl 0615.30005

[Douady and Hubbard 1985] A. Douady and J. H. Hubbard, "On the dynamics of polynomial-like mappings”, Ann. Sci. École Norm. Sup. (4) 18:2 (1985), 287-343. MR 87f:58083 Zbl 0587.30028

[Keen and Kotus 1997] L. Keen and J. Kotus, "Dynamics of the family $\lambda \tan z$ ", Conform. Geom. Dyn. 1 (1997), 28-57. MR 98h:58159 Zbl 0884.30019

[McMullen 2000] C. T. McMullen, "The Mandelbrot set is universal”, pp. 1-17 in The Mandelbrot set, theme and variations, edited by L. Tan, London Math. Soc. Lecture Note Ser. 274, Cambridge Univ. Press, 2000. MR 2002f:37081 Zbl 1062.37042

Received July 25, 2007.

Piotr GALĄZKa

FACULTY OF MATHEMATICS AND INFORMATION SCIENCES

WARSAW UNIVERSITY OF TECHNOLOGY

PlaC POLITECHNIKI 1

00661 WARSAW

POLAND

T.Galazka@mini.pw.edu.pl

JANINA KOTUS

FACULTY OF MATHEMATICS AND INFORMATION SCIENCES

WARSAW UNIVERSITY OF TECHNOLOGY

Plac Politechniki 1

00661 WARSAW

POLAND

J.Kotus@impan.gov.pl 